\title{
3D detectors
}

\section{Richard L. Bates ${ }^{* \dagger}$}

SUPA, School of Physics and Astronomy, Glasgow University, Glasgow, G12 8QQ, UK

E-mail: richard.bateseglasgow.ac.uk

The 3D detector was conceived as a method to overcome the radiation induced reduction in charge carrier lifetimes in heavily irradiated silicon detectors via the use of advanced MEMS device fabrication techniques. This paper reviews the state of the art in 3D detectors. Work performed within the major fabrication institutes will be discussed. This includes modifications to the original proposed design to reduce complexity and therefore increase device yield. The impact on performance of such modifications will be covered. Characterisation and performance of 3D detectors up to the maximum radiation fluence expected at the high luminosity LHC operation will be presented and compared to simulations. Results from both strip and pixel devices will be shown using characterisation methods that include 90-Sr Betas, focused laser and high-energy particle beams.

The 20th Anniversary International Workshop on Vertex Detectors - VERTEX 2011

June 19 - 24, 2011

Rust, Lake Neusiedl, Austria

\footnotetext{
${ }^{*}$ Speaker.

${ }^{\dagger}$ This work was supported in part by the UK Science and Technology Facilities Council, STFC.
} 


\section{Introduction}

To realise the full physics potential of the CERN Large Hadron Collider, (LHC), a series of planned luminosity upgrades are planned to the accelerator resulting in the High-luminosity LHC, (HL-LHC)[1, 2]. The HL-LHC will have an order of magnitude increase in integrated luminosity over the LHC to $10^{35} \mathrm{~cm}^{-2} \mathrm{~s}^{-1}$. To cope with the associated increase in event multiplicity and radiation damage the experiments based on the LHC will also undergo a series of upgrades. The expected maximum fluence inside the experiments will ultimately increase ten fold to maximum levels close to $10^{16} \mathrm{~cm}^{-2} 1 \mathrm{MeV}$ equivalent neutrons $(1 \mathrm{MeV} \mathrm{n}$ eq $)$ [3], consisting essentially of charged particles. Such doses are unprecedented for silicon detectors. To be able to cope with such high radiation levels new operating scenarios and detector designs are being investigated. One such detector design is the $3 \mathrm{D}$ detector.

\section{3D detector designs}

The 3D detector was originally proposed by Sherwood Parker[4] in 1997 as a method to overcome the reduced signal caused by the radiation induced reduction in the free carrier lifetime[5,6]. The 3D detector has an array of n- and p-type electrode columns passing through the silicon substrate rather than being implanted on its surface. These electrodes are realised by a combination of micro-machining techniques and standard detector technologies[7, 8]. This structure allows the combination of a standard substrate thickness with a lateral electrode spacing of a few tens of micrometers. As a result of the reduced electrode spacing, the depletion and charge collection distances are dramatically reduced, without reducing the sensitive thickness of the detector. This implies that the device has extremely fast charge collection and a low operating voltage, even after a high irradiation dose. The reduced operating voltage, compared to a planar device, results in lower power consumption in the 3D sensor. The short collection distance and the electric field pattern in the device also reduces the amount of charge trapping that takes place compared to a planar detector. These features should make 3D detectors substantially more radiation hard than standard detectors. The enclosed structure of the unit cell of the 3D detector will also reduce the amount of charge sharing that takes place. While this reduces the spatial resolution of the detector before irradiation, when signal magnitude is not an issue, the lack of charge sharing will increase the signal in a given pixel after heavy irradiation[9].

\subsection{Full 3D sensors}

The original 3D detector, known as the full 3D detector, is fabricated using one sided processing. This device is fabricated by first wafer bonding the sensor wafer to a support wafer. The holes for the electrodes are etched using a deep reactive ion etching based on the Bosh process. The first set of holes, for one electrode type, are etched through the sensor wafer and stop at the oxide interface with the support wafer. To form the electrode, these are filled with polysilicon, and doped, using low pressure chemical vapour deposition. After the first set of electrodes are fabricated the second set are etched and filled in the same fashion. The individual electrodes are connected together with metal deposited on the top surface to form pixel or strip detectors. Finally, the backside support wafer is removed. The full 3D detector allows, with relative ease, the addition 


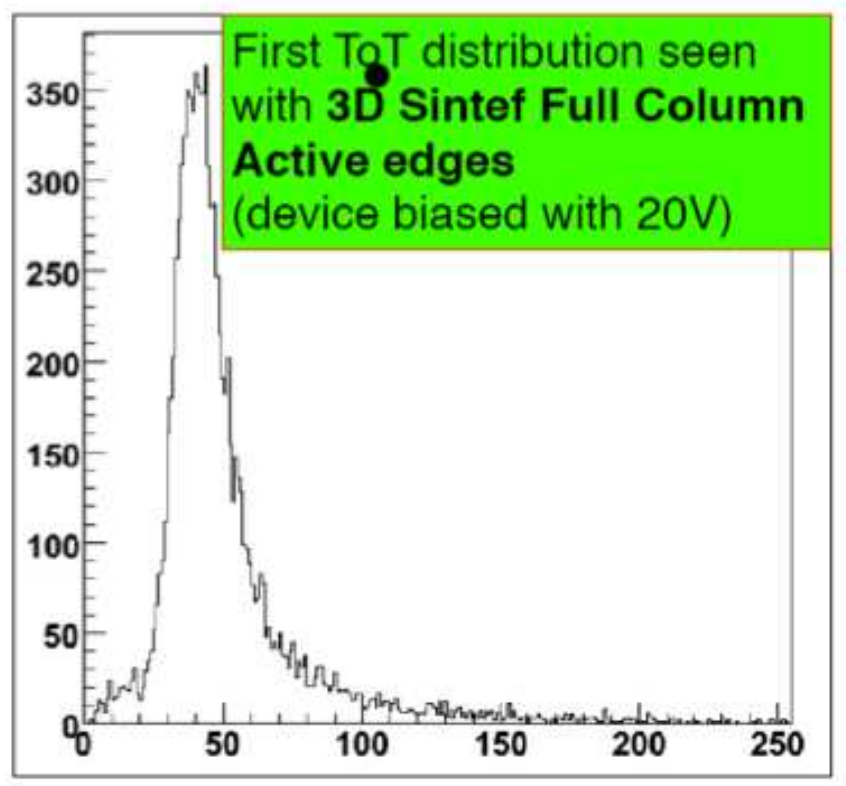

Figure 1: The pulse height spectra obtained from a full 3D detector fabricated by Sintef connected to an ATLAS FE-I3 pixel chip and illuminated by a 90-Sr source.

of an electrode around the full detector matrix, known as an active edge[10]. This contains the electric field and also allows the detector to be active all the way to the edge of the device. Full 3D detectors have been fabricated at both Sintef[11, 12] and Stanford[7].

Sensors that are compatible with the ATLAS FE-I3[13] front-end pixel amplifier chip have been fabricated at Sintef and successfully made into a module and tested at CERN. The pulse height spectra of a Sintef detector tested with a 90-Sr source is shown in Fig.1. The detector was biased to $20 \mathrm{~V}$ to over deplete it; full depletion was at $15 \mathrm{~V}$ according to electrical measurements.

The carrier lifetime in the polysilicon, in the filled column, should be sufficient to collect significant charge from radiation incident upon the column. However, studies with a fine focused $\mathrm{x}$-ray beam have shown that there is charge loss in the columns. The reason for this is that during the fabrication process an oxide is formed on the edge of the column which forms a barrier to carrier collection from the column[14]. A change in the dopant chemistry from $\mathrm{POCl}_{3}$ to $\mathrm{PH}_{3}$ for the n-type column and $\mathrm{BBr}_{3} / \mathrm{O}_{2}$ to $\mathrm{B}_{2} \mathrm{H}_{6}$ for the $\mathrm{p}$-type column should remove the oxygen trapping of the charge carries in the column and therefore increase charge collection from inside the column.

\subsection{Double-sided 3D detectors}

The second method to fabricated the 3D detector is known as the double-sided process which was introduced to remove the complexity of the support wafer. This method is being developed by CNM, Barcelona[8] and FBK, Trento[15]. The double-sided process, as the name suggests, utilizes double-sided alignment techniques to align the first set of etched holes with the second. The first set of holes are etched into the sensor wafer and fully, or partially filled, in the same fashion as with the full 3D detector. The sensor wafer is then processed on the back side to form the second set of holes. The holes may pass all the way through, or only part way through, the sensor wafer. 
The reduced fabrication complexity has increased the yield of the 3D device. However, if the columns do not penetrate the full thickness of the wafer, the sensor performance is sensitive to the penetration depth of the columns and this needs to be well controlled.

For a double-sided 3D detector with non-fully penetrating electrodes there is a lower field region directly above a column which requires the detector to be over depleted to be fully active[16]. The devices therefore show a two stage depletion process: at first a lateral depletion between the columns at low voltage, (for example $4 \mathrm{~V}$ ), followed by depletion from the column tips to the device surface at higher voltages, (for example $40 \mathrm{~V}$ ).

If the columns are only partially filled with polysilicon they are not themselves active, however these are easier to fabricated than filled columns and lead to increased yield in the device.

A further complication of the double-sided 3D detector is the lack of an active edge. To minimise the dead region at the edge of the sensor the guard fence structure has been developed[17]. The guard fence consists of multiple ohmic columns around the active silicon area. The ohmic columns stop the depletion region spreading from the pixel to the cut edge. With an ohmic column spacing of $50 \mu \mathrm{m}$ in the guard fence, it has been shown that the cut edge can be as close as $100 \mu \mathrm{m}$ from the active silicon without adversely affecting the current-voltage characteristics of the device.

\section{ATLAS IBL campaign}

Both the double-sided 3D and planar silicon detectors have been selected to be used for the first upgrade to the ATLAS silicon detector system, namely the insertable B-layer[18], (IBL). Both FBK and CNM are processing sensors for this experiment using a mask set that is as common as possible. Pixel sensors compatible with the ATLAS FE-I4[19] front-end pixel amplifier chip have been fabricated and extensively tested both before and after irradiation. The sensors show a good uniformity over the matrix for threshold, noise and detection response. A noise performance of 150 electrons at a threshold of 3200 electrons have been routinely obtained before irradiation. After an irradiation dose of $5 \times 10^{15} \mathrm{~cm}^{-2} 1 \mathrm{MeV} \mathrm{n}_{e q}$, from reactor neutrons, the module threshold and noise performance is only slightly altered, with a noise increase of only 5 electrons to 155 electrons, when measured at $-15^{\circ} \mathrm{C}$. The current of the device increased as expected from the leakage current damage factor in silicon.

\section{3D detector test beam analysis}

Many test beams have been performed on 3D detector modules. This paper will concentrate on the results from a test beam performed with a high energy pion beam from the CERN Super Proton Synchrotron (SPS). The 3D detector was fabricated at CNM and is a double-sided device. It was fabricated on 4 inch float zone silicon wafers with a resistivity of $13 \mathrm{k} \Omega \mathrm{cm}$. The columns have a diameter of $10 \mu \mathrm{m}$, with a depth of $250 \mu \mathrm{m}$ in a $285 \mu \mathrm{m}$ thick substrate. The devices are hole collecting with an n-type substrate and p-doped columns connected to the electronic readout. The devices were solder bump-bonded to a Timepix[20] readout chip. Using current-voltage and capacitance-voltage techniques, lateral depletion was measured at $2 \mathrm{~V}$ and full depletion at $10 \mathrm{~V}$. The device reported here was operated over depleted at $20 \mathrm{~V}$, where it had a leakage current of $3.8 \mathrm{~mA}$ at room temperature. 


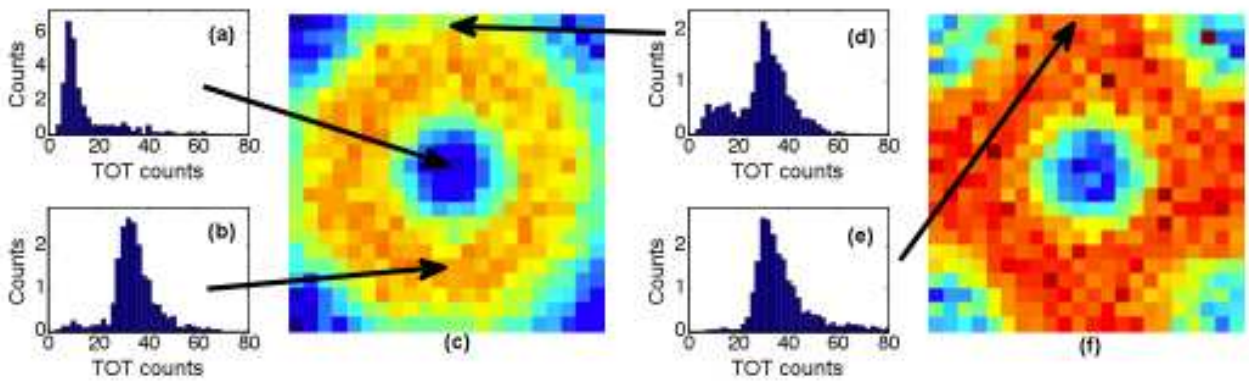

Figure 2: The pulse height spectra as a function of incident position, obtained from a double-sided 3D detector fabricated by CNM connected to a TimePix pixel chip and illuminated by a high energy pion beam. The histograms are of the ToT counts in the central electrode region (a), away from the central electrode and pixel edges (b). Pixel maps showing the mean energy deposition across the pixel matrix, for a single pixel (c) and the energy in clusters (f). (d) and (e) show the histograms of the energy deposited at the pixel edges for the single pixel and the clusters.

The 3D detector assembly was placed on a precision $x-y$-theta stage at the centre of a beam telescope made from 6 TimePix planar silicon detector assemblies. The telescope has a pointing resolution for reconstructed tracks at the device under test of $2.3 \pm 0.1 \mu \mathrm{m}[21]$.

The energy collected in the pixel unit cell was reconstructed as a function of incident position of the pion beam, as shown in Fig. 2. The charge collected for pions incident on the pixel sensor in the region distant from the columns showed a pulse height spectra well described by a landau function. The most probably value obtained was that expected from a $285 \mu \mathrm{m}$ thick silicon detector. Pions incident at the edge of the pixel cell exhibited charge sharing into the neighbouring pixel, manifested as a low energy peak in the pulse height spectra shown in Fig.2d. When the energy deposited in the neighbouring pixel is combined into a cluster, the cluster shows full charge collection all the way to the edge of the pixel cell, see Fig.2e. The centre of the pixel cell shows full charge collection from the silicon above the column, which is $35 \mu \mathrm{m}$ thick. The charge collected at the corner of the pixel is low as the charge collected in the $35 \mu \mathrm{m}$ of silicon above the ohmic column is shared between four pixels and falls below the pixel threshold.

The average detection efficiency of the full pixel was found to be $93 \%$ for normal incidence due to the low efficiency in the corners of the pixel unit cell. As the angle of incidence was increased to 10 degrees the detection efficiency increased to $99.8 \pm 0.5 \%$ across the pixel matrix. At an angle of 10 degrees the track traverses a full pixel within the thickness of the sensors. This is also the angle at which the best spatial resolution is obtained of $9.18 \pm 0.1 \mu \mathrm{m}[21]$. At normal incidence the spatial resolution is $15.8 \pm 0.1 \mu \mathrm{m}$ which is in agreement with the binary resolution of the pixel which has a pitch of $55 \mu \mathrm{m}$. This is due to the low charge sharing present in the 3D detector structure. More details of the test beam analysis can be found in [22].

\section{Charge collection in $3 D$ detectors}

Short $285 \mu \mathrm{m}$ thick 3D strip sensors have been fabricated to study charge collection in the 3D sensor after heavy irradiation. The short strip device was chosen as it decouples the electronics 


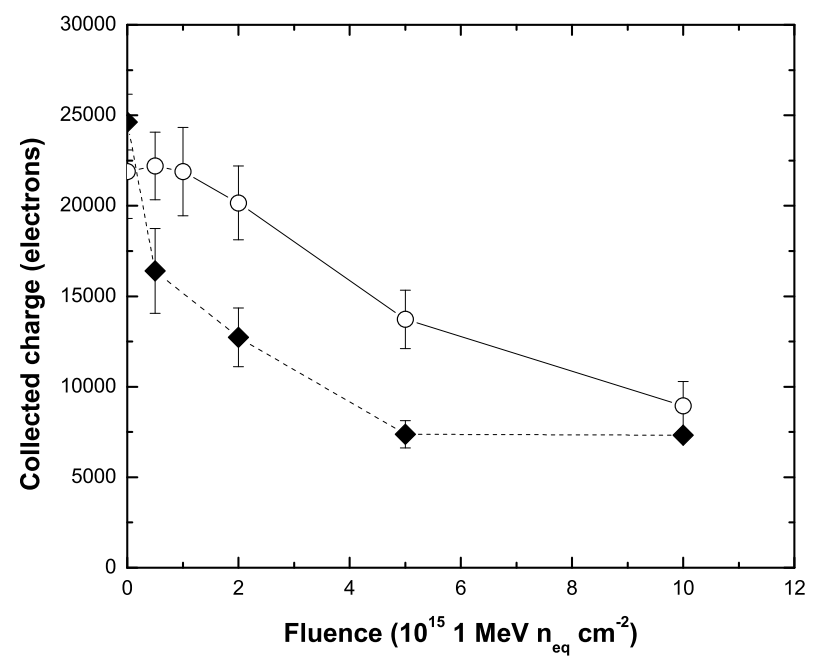

Figure 3: The charge collection as a function of fluence for a $285 \mu \mathrm{m}$ thick 3D sensor operated at $150 \mathrm{~V}$ (open circles) and a $300 \mu \mathrm{m}$ thick planar sensor operated at $1000 \mathrm{~V}$ (closed diamonds).

from the sensor during the irradiation period. The short strip device has an $80 \mu \mathrm{m}$ pitch between columns of the same type and therefore strips of a pitch of $80 \mu \mathrm{m}$.

The sensors have been irradiated up to a fluence of $2 \times 10^{16} \mathrm{~cm}^{-2} 1 \mathrm{MeV} \mathrm{n}$ eq. They were tested with a 90-Sr source and readout with the LHC speed analogue front-end Beetle chip[23], which was part of the Alibava data acquisition system[24]. The systems were placed in a freezer and the temperature of the ceramics, on which the devices under test were mounted, were kept at $-13.4 \pm 2.2^{\circ} \mathrm{C}$. For a full description of the set-up including gain calibrations please see [25].

The results of the charge collected as a function of fluence are shown in Fig.3, for a sensor bias voltage of $150 \mathrm{~V}$. The $3 \mathrm{D}$ detector gives full charge collection up to a fluence of $10^{15} \mathrm{~cm}^{-2}$ $1 \mathrm{MeV} \mathrm{n}_{e q}$, which falls to $47 \%$ after a fluence of $10^{16} \mathrm{~cm}^{-2} 1 \mathrm{MeV} \mathrm{n}_{e q}$. The noise remains constant as a function of fluence and therefore the signal-to-noise ratio as a function of fluence follows the collected charge curve. The collected charge in the 3D detector is well modelled by TCAD simulation without any high field effects being required. The signal is more than that observed in a $300 \mu \mathrm{m}$ thick planar device operated at a bias voltage of $1000 \mathrm{~V}$, which collected $30 \%$ of the expected deposited charge after a fluence of $10^{16} \mathrm{~cm}^{-2} 1 \mathrm{MeV} \mathrm{n}$ eq. The larger signal in the 3D detector is due to the higher electric fields inside the 3D sensor and the shorter collection distances compared to the planar device.

The collected charge in the 3D detector increases greatly with an increase in bias voltage to values up to $350 \mathrm{~V}$, this is shown in Fig.4. The increase in charge is due to charge multiplication, which is evident for devices irradiated to less than $2 \times 10^{15} \mathrm{~cm}^{-2} 1 \mathrm{MeV} \mathrm{n} e q$. The multiplication is believed to be due to impact ionisation which takes place in the high field regions around the electrodes, which extend through the entire device thickness. At these higher bias voltages a charge of $50 \%$ of that expected is collected after a fluence of $2 \times 10^{16} \mathrm{~cm}^{-2} 1 \mathrm{MeV} \mathrm{n} e q$. Full details of the charge collection studies can be found in [25]. 


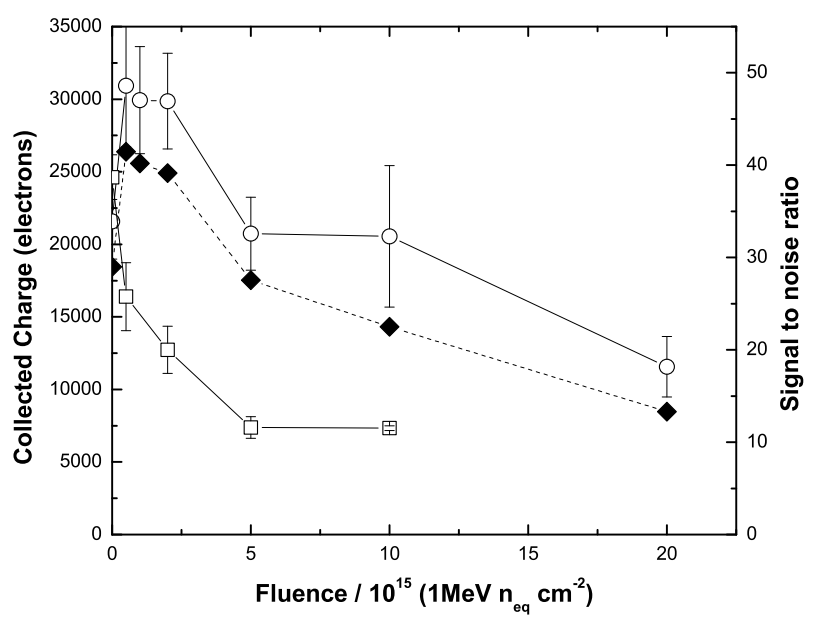

Figure 4: The charge collection as a function of fluence for a $285 \mu \mathrm{m}$ thick $3 \mathrm{D}$ sensor operated at 250 to $350 \mathrm{~V}$ (open circles) and a $300 \mu \mathrm{m}$ thick planar sensor operated at $1000 \mathrm{~V}$ (open squares). The signal-tonoise ratio of the $3 \mathrm{D}$ sensor is also shown (closed diamonds).

Infra-red light of $974 \mathrm{~nm}$ in wavelength from a laser diode was focused, with a spot size of $4 \mu \mathrm{m}$, on the surface of the 3D strip sensors. The light source was scanned across the device under test with precision $\mathrm{x}-\mathrm{y}$ stages in $2 \mu \mathrm{m}$ steps. The sensor was readout with the Alibava system. From the relative signal collected a response map of the device was built up for a unit cell. The spatially resolved laser scanning showed a uniform response across the unit cell outside the column regions for an un-irradiated sensor when operated above full depletion. After heavy irradiation, $\left(2 \times 10^{15} \mathrm{~cm}^{-2} 1 \mathrm{MeV} \mathrm{n}_{e q}\right)$, the response was non-uniform with an area of low charge being collected in regions of low field between columns of the same doping type and an enhanced signal in the regions of high field between columns of different doping, as shown in Fig.5. The enhanced collection in the high field regions is assumed to be due to charge multiplication in the high field regions close to the junction electrodes.

Simulations have been performed using TCAD. The Perugia[26] model for the radiation induced defects were included and the high field effects of impact ionisation and band-to-band tunnelling have been used. A charge package was introduced that represents the charge deposited by a minimum ionising particle inside the device. A transient simulation was run and the signal induced on the collecting electrode recorded. For a device, with defects appropriate to a fluence of $2 \times 10^{15} \mathrm{~cm}^{-2} 1 \mathrm{MeV} \mathrm{n}_{e q}$, charge multiplication was observed. When the charge was deposited at the high field region between columns of different doping types, and a bias of $250 \mathrm{~V}$ applied to the detector, the collected charge was 1.8 times that deposited.

\section{Conclusions}

3D silicon detectors are now well developed. The full 3D and the double-sided 3D detector can both be fabricated in industry. The double-sided device to date has a better yield due to the 


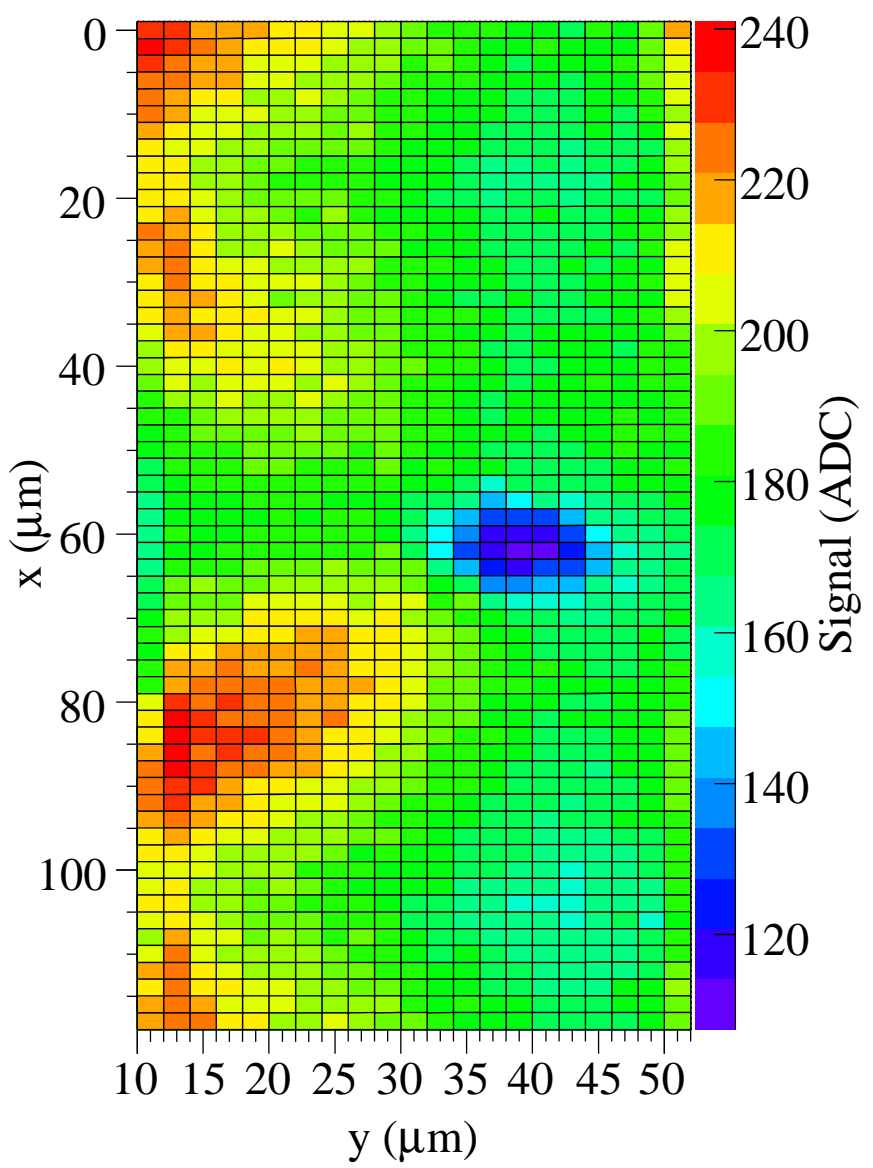

Figure 5: The response of the $3 \mathrm{D}$ detector biased to $260 \mathrm{~V}$ to collimated laser light after a fluence of $2 \times 10^{15} \mathrm{~cm}^{-2} 1 \mathrm{MeV} \mathrm{n}_{e q}$. The juntion columns are at $(\mathrm{x}, \mathrm{y})$ positions $(20,0)$ and $(100,0)$ and the ohmic column is at $(60,40)$. The metal strip connecting the junction columns is present from $\mathrm{y}=-10$ to $\mathrm{y}=10$ and prevents data being collected in this area.

less demanding processing technology. The double-sided 3D detector has been chosen as a sensor solution for the first upgrade to the ATLAS silicon system, namely the IBL.

Precision scans of the pixel have been preformed in high energy test beams. The charge deposition in a unit cell has been mapped and full charge collection is observed in the majority of the cell. The charge collected above the central column is consistent with full charge collection from the $35 \mu \mathrm{m}$ of silicon present at this location. A high detection efficiency across the pixel matrix of $93 \pm 0.5 \%$ at zero degrees incident angle has been observed. This increases to $99.8 \pm 0.5 \%$ at an angle of incidence of 10 degrees. The charge sharing has been shown to be less than for a planar device resulting in lower spatial resolution, (consistent with binary readout), but this might result in more charge being collected in the hit pixel after irradiation.

The charge collected in a laboratory $90-\mathrm{Sr}$ source test shows higher charge collected in the 3D detector compared to the planar device. After an irradiation dose of $10^{16} \mathrm{~cm}^{-2} 1 \mathrm{MeV} \mathrm{n}_{e q}$, a 3D 
detector operated at a modest bias voltage of $150 \mathrm{~V}$ collected $47 \%$ of the deposited charge compared to $30 \%$ for the planar device operated at $1000 \mathrm{~V}$. At higher bias voltages, charge multiplication was observed in the 3D irradiated device. Spatially resolved laser scanning showed a uniform response across the unit cell outside the column region for an un-irradiated sensor when operated above full depletion. After heavy irradiation the response was non-uniform with an area of low charge being collected in regions of low field between columns of the same doping and an enhanced signal in the region of high field between columns of different doping. Simulations have been able to predict charge multiplication in the 3D sensor.

\section{References}

[1] F. Ruggiero, LHC accelerator R\&D and upgrade scenarios, Eur. Phys. J. C, vol. 34, no. 1, pp. 433 - 442, 2004.

[2] F. Gianotti, et al., Physics potential and experimental challenges of the LHC luminosity upgrade, Eur. Phys. J. C, vol 39, no. 3, pp. 293 - 333, 2005.

[3] I. Dawson, Atlas Tracker Upgrade Workshop, December 2007, Valencia. http://indico.cern.ch/conferenceDisplay.py?ovw=True \& confId=21398.

[4] S. I. Parker, C. J. Kenney, J. Segal, 3D - a proposed new architecture for solid-state radiation detectors', Nucl. Instr. and Meth A, vol. 395, pp. 328 - 343, 1997.

[5] G. Kramberger, V. Cindro, I. Mandic, M. Mikuz and M. Zavrtanik, Determination of effective trapping times for electrons and holes in irradiated silicon, Nucl. Instr. and Meth. A, vol. 476, pp. 645 - 651, 2002.

[6] A. G. Bates and M. Moll, A comparison between irradiated magnetic Czochralski and float zone silicon detectors using the transient current technique, Nucl. Instr. and Meth. A, vol. 555, pp. 113 - 124, 2005.

[7] C. Kenney, S. Parker, J. Segal, C. Storment, Silicon detectors with 3-D electrode arrays: fabrication and initial test results, IEEE Trans. Nucl. Sci., vol. 46, no. 4, pp. 1224 - 1236, 1999.

[8] G. Pellegrini et al., First double-sided 3-D detectors fabricated at CNM-IMB, Nucl. Instr. and Meth. A, vol. 592, pp. 38 - 43, 2008.

[9] C. DaVia, S. J. Watts, The geometrical dependence of radiation hardness in planar and 3D silicon detectors, Nucl. Instr. and Meth. A, vol. 603, pp. 319 - 324, 2009.

[10] C. J. Kenney, S. Parker, E. Walckiers Results from 3-D silicon sensors with wall electrodes: near-cell-edge sensitivity measurements as a preview of active-edge sensors, IEEE Trans. Nucl. Sci., vol. 48, no. 6, pp. 2405 - 2410, 2001.

[11] T. E. Hansen, et al., First fabrication of full 3D-detectors at SINTEF, JINST, 4 (2009) P03010. doi:10.1088/1748-0221/4/03/P03010.

[12] A. Kok, et al., Fabrication of 3D silicon sensors, Proceedings of Science, Vertex 2010, Paper 022, 2010.

[13] I. Peric, et al., The FEI3 readout chip for the ATLAS pixel detector, Nucl. Inst. and Meth. A, vol. 565, pp. 178 - 187, 2006.

[14] J. Hasi, Status of 3D sensors processing at STANFORD, The $5^{\text {th }}$ "Trento" workshop on advanced silicon radiation detectors. 
[15] A. Zoboli et al., Double-Sided, Double-Type-Column 3-D Detectors: Design, Fabrication, and Technology Evaluation, IEEE Trans. Nucl. Sci., vol. 55, no. 5, pp. 2775 - 2284, 2008

[16] D. Pennicard, et al., Simulation results from double-sided 3-D detectors, IEEE Trans. Nucl. Sci., vol. 54, no. 4, pp. 1435 - 1443, 2007.

[17] G. D. Betta, et al., Development of modified 3D detectors at FBK, 2010 IEEE Nuclear Science Symposium, Knoxville (USA), Oct. 30 - Nov. 6, 2010, Conference Record, Paper N15-3.

[18] P. Sicho, SLHC upgrade plans for the ATLAS pixel detector, Nucl. Instr. and Meth. A, vol. 607, pp. $31-34,2009$.

[19] M. Garcia-Sciveres, et al., The FE-I4 pixel readout integrated circuit, Nucl. Instr. and Meth. A, vol. 636, pp. 155 - 159, 2011

[20] X. Llopart et al., Timepix, a 65k programmable pixel readout chip for arrival time, energy and/or photon counting measurements, Nucl. Instr. and Meth. A, vol. 581, pp. 485 - 494, 2007.

[21] A. Kazuyoshi et al., Charged particle tracking with the Timepix ASIC, Nucl. Instr. and Meth. A, In Press, doi 10.1016/j.nima.2011.09.021.

[22] A. MacRaighne et al., Precision scans of the Pixel cell response of double sided 3D Pixel detectors to pion and X-ray beams, JINST, 6 (2011) P05002. doi:10.1088/1748-0221/6/05/P05002.

[23] The Beetle Manual Reference, CERN, CERN-LHCb-2005-105.

[24] R. Marco-Hernández and ALIBAVA COLLABORATION, A Portable Readout System for Microstrip Silicon Sensors (ALIBAVA), IEEE Trans. Nucl. Sci., vol. 56, no. 3, pp. 1642 - 1649, 2009.

[25] R. Bates, Charge collection studies and electrical measurements of heavily irradiated 3D Double-Sided sensors and comparison to planar strip detectors, IEEE Trans. Nucl. Sci., To be published.

[26] M. Petasecca, F. Moscatelli, D. Passeri, G. U. Pignatel, Numerical Simulation of Radiation Damage Effects in p-Type and n-Type FZ Silicon Detectors, IEEE Trans. Nucl. Sci., vol. 53, no. 5, pp. $2971-2976,2006$ 